\title{
Hegemonia, gubernamentalidad, territorio. Apuntes metodológicos para una historia social de la planificación
}

\author{
Hegemony, governmentality, territory. Methodological notes on \\ the social history of planinng
}

\author{
Álvaro Sevilla Buitrago \\ Universidad Politécnica de Madrid \\ alvaro.sevilla@upm.es
}

Recibido: 9.102012

Aceptado: 6.11 .2013

\section{RESUMEN}

La historia social de la planificación espacial es un elemento clave en el proyecto de replanteamiento crítico de las políticas urbanas y territoriales. Un urbanismo comprometido con la recuperación de lo común bajo coordenadas radicalmente democráticas requiere una lectura del pasado capaz de desvelar cómo llegaron a producirse las estructuras socioespaciales contemporáneas y el papel que la planificación jugó en dicho proceso. La historia social de la planificación permite comprender la génesis sociopolítica de los discursos, técnicas y prácticas urbanísticas que empleamos en la actualidad y cuál ha sido su efecto sobre la vida cotidiana de los planificados. Este artículo analiza varios momentos conceptuales y metodológicos de ese proyecto historiográfico. Se exploran en primer lugar el concepto gramsciano de «hegemonía» y el foucaultiano de «gubernamentalidad» como herramientas que permiten comprender la articulación entre política y vida cotidiana en el marco de una historia social general. Esta matriz de trabajo se traslada a la dimensión espacial a través de los conceptos de «territorio» y «territorialidad». Por último se estudia el modo en que la planificación ha sido movilizada históricamente como técnica de regulación espacial de los procesos de reproducción social por determinados proyectos hegemónicos/ gubernamentales en un contexto de lucha de territorialidades.

\section{PALABRAS CLAVE}

Reproducción social, hegemonía, gubernamentalidad, vida cotidiana, territorialidad.

EMPIRIA. Revista de Metodología de Ciencias Sociales. N. ${ }^{\circ}$ 27, enero-abril, 2014, pp. 49-72. ISSN: 1139-5737 DOI 10.5944/empiria.27.10862 


\begin{abstract}
The social history of spatial planning is a key element in the critical reevaluation of urban and territorial policies. In order to engage planning in the recovery of the commons and the formation of more democratic environments we need to understand its role in the historical production of our present sociospatial structures. This historiographical approach provides an alternative account of the sociopolitical genesis of contemporary planning discourses, techniques and practices, describing their effects and impact on the everyday lives of planned populations. This article analyzes several conceptual and methodological moments of this research project. Firstly I suggest that we use Gramsci's conceptualization of «hegemony « and Foucault»s «governmentality» in order to understand the articulation between politics and everyday life in the context of a general social history. This theoretical framework is then translated into spatial terms through the concepts of «territory» and «territoriality». Finally, I study how planning has been mobilized throughout history by particular hegemonic/governmental projects in order to spatially regulate the processes of social reproduction, in a context shaped by struggles of territorility.
\end{abstract}

\title{
KEYWORDS
}

Social reproduction, hegemony, governmentality, everyday life, territoriality.

\section{INTRODUCCIÓN}

Las técnicas y los saberes no son inocentes. Evaluar críticamente el modo en que una disciplina ha sido interpelada por determinados entramados de poder y cómo dicha articulación se proyecta sobre la vida de los ciudadanos es un requisito indispensable para comprometerla socialmente (Althusser 1988; Pickering 1995; Barnes 2012). En el caso de la planificación espacial esta tarea exige una serie de movimientos teóricos previos. En primer lugar, es necesario integrar el momento espacial en un marco de teoría social y política más amplio - examinar social y políticamente el espacio, analizar espacialmente lo social y lo político (Dikeç 2012). Como sugiere Doreen Massey: «Pensar lo espacial ... permite replantear el modo en que se formulan ciertas cuestiones políticas, puede servir de ayuda en las controversias ya abiertas y, sobre todo, puede convertirse en un elemento esencial en la estructura imaginativa que permite ... una apertura de la propia esfera de lo político» (Massey 2005: 9). En segundo lugar, es necesario comprender cuál es la relación entre lo espacial y la vida cotidiana, cómo median en esa relación las técnicas de regulación del espacio y cuáles son los efectos y resultados que podemos esperar cuando dichas técnicas operan en la órbita de lo que aquí denominaremos proyectos de gobierno de los procesos de reproducción

EMPIRIA. Revista de Metodología de Ciencias Sociales. N. ${ }^{\circ}$ 27, enero-abril, 2014, pp. 49-72. ISSN: 1139-5737 DOI 10.5944/empiria.27.10862 
social $^{1}$. En síntesis, una crítica de la razón de poder — de la gubernamentalidad - de la planificación debe comprender la conexión que existe, por un lado, entre política, espacio y planificación, y, por otro, entre espacio, planificación y vida cotidiana; o, de modo más sintético, explorar el modo en que la planificación espacial media en el proyecto de gobierno de los procesos de reproducción social a través de la producción de territorios y territorialidades.

En este trabajo presentaré la historia social de la planificación como uno de los movimientos epistemológicos que pueden ayudarnos en ese empeño y exploraré de forma tentativa algunos de sus momentos conceptuales y metodológicos. ¿De qué modo puede contribuir esta nueva historiografía en el empeño que acabo de formular? Comprendida como «historia del presente» - como arqueología de los elementos que componen las estructuras sociales contemporáneas ${ }^{2}$ - una historia social de la planificación nos permite comprender la génesis sociopolítica de los discursos, técnicas y prácticas urbanísticas que empleamos en la actualidad y cuál ha sido su efecto sobre la vida cotidiana de los «planificados», las poblaciones reordenadas espacialmente por la acción de la planificación a lo largo del tiempo. El planificador contemporáneo ignora con frecuencia la historia localmente específica de esos recursos y herramientas, útiles de trabajo que ha heredado como una forma vacía de contenido en la que a menudo se oculta, naturalizado en la práctica, un pasado inhabilitante. La historia social de la planificación proporciona la oportunidad para subsanar dichas lagunas, para corregir las carencias y aspectos socialmente regresivos de los instrumentos de planificación y reinventar su cometido con principios emancipadores. En suma, esta forma de escribir la historia de las políticas espaciales nos permite comprender y evaluar críticamente las deudas históricas de la planificación como paso previo a su subsanación, en el marco de un proyecto más amplio para comprometer sus técnicas en un horizonte de equidad y justicia socio-espacial.

Este artículo esboza algunos de los supuestos metodológicos de esta empresa historiográfica. En el siguiente apartado exploraré ciertos conceptos y pautas de investigación que, desde la perspectiva de una historia social gene-

1 Adoptamos aquí una noción amplia del concepto de «reproducción social». Más allá de las restricciones fijadas en el esquema dual del marxismo ortodoxo —que separaba producción y reproducción social, reservando a la segunda un papel subordinado de complemento no determinante de los procesos de cambio social- y recogiendo las aportaciones de sucesivas oleadas de teoría social, entendemos integrados en los procesos de reproducción social los aspectos relativos a la existencia y la vida cotidiana, la producción y cuidado de la fuerza de trabajo, los códigos del consumo, del tiempo libre, los procesos de socialización y comunicación y la mediación institucional de los mismos, la replicación de estructuras sociales segregadas y jerárquicas, las políticas de la identidad, la producción de memoria e imaginarios colectivos, etc.; o, en términos más cercanos al discurso de la planificación, la proyección de todos estos procesos sobre la ciudad, los soportes espacio-temporales que perfilan una concreta economía política del cuerpo y sus comportamientos, de la experiencia individual y colectiva, de las relaciones socioespaciales.

2 Mitchell Dean ha caracterizado la historia del presente que Foucault (1975:35) sugiriera en Vigilar y castigar como un intento de problematizar «lo que se da por hecho, lo que se asume como dado o natural en la existencia social contemporánea, una naturalidad ... que se ve cuestionada en el curso de las luchas actuales» (Dean 1994:35).

EMPIRIA. Revista de Metodología de Ciencias Sociales. N. ${ }^{\circ}$ 27, enero-abril, 2014, pp. 49-72. ISSN: 1139-5737 DOI 10.5944/empiria.27.10862 
ral, permiten conectar con eficacia las instancias de la política convencional y la vida cotidiana. A tal efecto se analizarán las oportunidades implícitas en la articulación de los conceptos de «hegemonía» y «gubernamentalidad», en la formulaciones originales que les dieron Antonio Gramsci y Michel Foucault respectivamente; se examinarán sus puntos en común así como sus diferencias y carencias, proponiendo una integración-superación de las mismas a través del estudio histórico de la vida cotidiana, de su papel en los procesos de reproducción social y su objetivación en el marco de proyectos políticos más amplios. En el tercer apartado analizaré la relación existente entre el espacio, la ciudad y los procesos de reproducción social. Siguiendo la formulación de ciertas corrientes de geografía crítica, sugeriré que los conceptos de «territorio» $\mathrm{y}$ «territorialidad» resultan más precisos para comprender el carácter procesual, relacional y político de la producción social del espacio; tomando una particular aproximación a estos términos, indicaré que éstos proporcionan una espacialización efectiva de las categorías de «hegemonía» y «gubernamentalidad», según se han definido en el apartado anterior. A la luz de lo expuesto hasta ese momento, el cuarto apartado entiende la planificación como un dispositivo de gobierno encargado de regular espacialmente los procesos de reproducción social, de desterritorializar y reterritorializar los patrones de vida cotidiana incompatibles con sucesivos nuevos órdenes socioespaciales a lo largo del tiempo. A pesar del carácter histórica y geográficamente específico de estos órdenes, patrones y procesos - así como de las propias herramientas, modelos y discursos que la planificación despliega - puede rastrearse en ese ejercicio de regulación una lógica continua de desposesión de los capitales territoriales de los grupos sociales subalternos, de su capacidad para apropiarse del espacio, organizarlo, representarlo e imaginarlo. Por último, las conclusiones esbozan el modo en que esta historia social de la planificación puede contribuir en el proyecto más amplio de recuperación de espacios comunes bajo coordenadas radicalmente democráticas.

\section{POLÍTICA Y VIDA COTIDIANA: LOS CONCEPTOS DE «HEGEMONÍA»Y «GUBERNAMENTALIDAD» EN LA HISTORIA SOCIAL}

Como ha señalado Simon Gunn la labor historiográfica ha evolucionado en las últimas décadas siguiendo un hilo no tanto «vinculad[o] a nuevos hallazgos empíricos sino a una serie de momentos teóricos ... a conjunciones de historia y teoría» (Gunn 2011: 10). En el presente apartado exploraré algunas de esas conjunciones, en particular aquellas que considero más fructíferas para esbozar un método de investigación propio para la historia social de la planificación. Como he adelantado, este proyecto pretende comprender la articulación de las técnicas territoriales con estrategias políticas más amplias y sus proyectos de orden social; en otras palabras, describe la evolución de la planificación como una función de la regulación espacial de la reproducción

EMPIRIA. Revista de Metodología de Ciencias Sociales. N. ${ }^{\circ}$ 27, enero-abril, 2014, pp. 49-72. ISSN: 1139-5737 DOI 10.5944/empiria.27.10862 
social. Esta tarea requiere una conceptualización compleja de lo político, atenta a las conexiones entre el Estado y los espacios de la vida cotidiana, al modo en que el poder circula más allá de las instituciones, percolando a través de la totalidad del espacio social. El propio Gunn ha identificado en otro lugar dos de los principales paradigmas teóricos a través de los cuales la historia social general se ha enfrentado a ese reto: el cuadro conceptual proporcionado por la noción gramsciana de «hegemonía» y el derivado del trabajo de Michel Foucault en torno a la idea de «gubernamentalidad» (Gunn 2006). Aunque estos marcos de referencia pueden ser presentados como episodios sucesivos en la interacción reciente entre teoría e historia social, merece la pena establecer un diálogo entre ambos con el fin de perfeccionar y matizar el análisis que pueda resultar de su aplicación. Este eclecticismo se inscribiría en el proyecto más amplio de combinación de las perspectivas marxista y foucaultiana para una comprensión más integral de las dinámicas históricas y políticas (Jessop 2001; MacDonald 2002), en particular con vistas a la producción de una geografía histórica de las configuraciones de poder y su proyección sobre la población (Driver 1985; Sevilla-Buitrago 2012). Se trata, desde luego, de un eclecticismo arriesgado ${ }^{3}$, pero como intentaré demostrar los resultados potenciales justifican plenamente este matrimonio a priori ilícito.

Son muchas las semejanzas entre los acercamientos de Gramsci y Foucault a sus respectivas categorías de «hegemonía» y «gubernamentalidad» - con toda seguridad más de las que sus respectivas escuelas estarían dispuestas a reconocer. En su caracterización de la supremacía de los bloques históricos, Gramsci (2007: 2010-1) deslinda dos funciones: el «dominio» y la «dirección intelectual y moral» ${ }^{4}$. Cada uno de estos momentos políticos o formas de poder se ejerce fundamentalmente sobre dos esferas y a través de mecanismos diversos. El dominio, como labor de gobierno, se ejecuta desde las instituciones estatales a través de mecanismos coercitivos, fundamentalmente los asociados a la justicia, la labor policial y el ejército. La dirección intelectual y moral, la hegemonía propiamente dicha, se despliega por el contrario en la esfera de la sociedad civil, más allá del Estado y, especialmente, al margen de sus aparatos represivos. La hegemonía aparece como un principio de dirección en toda una serie de ámbitos (económico, intelectual, moral...) que permite a un determinado bloque social guiar la sociedad a través del consenso y sin imposiciones violentas, lo que garantiza un orden político estable y duradero. Ciertos matices del concepto resultan de especial interés para comprender el modo en que la propuesta gramsciana permite leer las conexiones entre política institucional

3 Especialmente teniendo en cuenta que ambos conceptos, «hegemonía» y «gubernamentalidad», encontraron sus desarrollos más ambiciosos en textos que no fueron elaborados para su publicación - los Quaderni del Carcere y los apuntes para los cursos en el College de France de 1977-1978 (Foucault 2008) y 1978-1979 (Foucault 2009), respectivamente- lo que a menudo dificulta los intentos de comprensión sistemática de los mismos.

4 Para un análisis del concepto de hegemonía ver Williams (1960), Bates (1975), Femia (1979), Urbinati (1998), además de la revisión y proyección desarrollada en Laclau \& Mouffe (1987).

EMPIRIA. Revista de Metodología de Ciencias Sociales. N. ${ }^{\circ}$ 27, enero-abril, 2014, pp. 49-72. ISSN: 1139-5737 DOI 10.5944/empiria.27.10862 
y vida cotidiana, así como para posibilitar un rastreo posterior de los paralelismos con la noción foucaultiana de gubernamentalidad:

a. Gramsci indica que la función hegemónica se despliega de modo «difuso», percolando todo el tejido social «[e]n forma y con medios que podemos llamar «liberales», es decir, a través de la iniciativa individual, «molecular», «privada» (esto es, no por un programa de partido elaborado y constituido según un plan que precede a la acción práctica y organizativa)» (Gramsci 2007: 2011). Podemos hablar por tanto de una «hegemonía liberal» que circula más allá de las instituciones estatales y afecta a los individuos inadvertidamente en su esfera privada.

b. La hegemonía desempeña una labor fundamentalmente pedagógica a muy diversos niveles, más allá de las estructuras de enseñanza oficiales. «Esta relación se da en la sociedad toda ... para cada individuo respecto a otros individuos, entre capas intelectuales y no intelectuales, entre gobernantes y gobernados, entre élites y aliados, entre dirigentes y dirigidos ... Toda relación de hegemonía es necesariamente una relación pedagógica» (Gramsci 2007: 1331).

c. Dicha labor formativa transmite una concepción del mundo que se integra en las conciencias como sentido común y se traslada al nivel de lo vivido en las prácticas materiales y el lenguaje. La concepción común del mundo permite alcanzar una unidad cultural-social que homogeneiza la diversidad de fines y valores de la población, manifestándose como un principio «general y particular, operante de forma transitoria - por vía emocional - o permanente, por [el] cual la base intelectual se haya tan enraizada, asimilada, vivida, que puede devenir pasión» (Gramsci 2007: 1331).

d. La finalidad última del momento hegemónico es la legitimación de un determinado proyecto de orden social, facilitando la labor de gobierno - el momento del dominio - mediante la producción de consenso y reduciendo por tanto la necesidad de coerción y uso de la fuerza. De ser necesario, el ejercicio de la fuerza «aparecerá apoyado en el consenso de la mayoría, expresado en la ... opinión pública» (Gramsci 2007: 1638), y se ejecutará sobre «aquellos grupos que no «consienten» activa ni pasivamente» (Gramsci 2007: 1519). Por tanto, la hegemonía prepara el terreno para la posterior labor de gobierno y minimiza, legitimándolo, el uso de la violencia. Se trata en suma de una forma de poder que se anticipa al conflicto social abierto, evitándolo.

Por su parte Michel Foucault ${ }^{5}$ identifica en su genealogía de la gubernamentalidad moderna una serie de momentos que habrían de orientarla hacia el control de la población y las subjetividades, en un sentido que recuerda a menudo

5 Para un análisis más detenido del concepto de «gubernamentalidad» ver Burchell et al. (1991), Dean (1999), Elden (2007).

EMPIRIA. Revista de Metodología de Ciencias Sociales. N. ${ }^{\circ}$ 27, enero-abril, 2014, pp. 49-72. ISSN: 1139-5737 DOI 10.5944/empiria.27.10862 
la aproximación gramsciana. Esta nueva racionalidad de gobierno «pretendió hacerse cargo de la conducta de los hombres» (Foucault 2008: 194), despuntando en el período comprendido entre finales del s. XVI y el s. XIX. Dos episodios tienen especial influencia en esta genealogía, según Foucault. En primer lugar el «pastorado» —en particular, el pastorado cristiano- entendido como oikonomia psychon, como conducta de las almas, «un poder ejercido sobre una multiplicidad y no sobre un territorio ... que guía hacia una meta [y] apunta a la vez a todos y a cada uno [de los pastoreados]» (Foucault 2008: 135). Foucault identifica en esta configuración el preludio al desplazamiento del territorio a la población como principal objeto del poder en la gubernamentalidad moderna, un esquema inicial de su «conducta de las conductas». El segundo episodio clave al que alude Foucault es el estado policial de los siglos XVI-XVII, primera forma de estado gubernamental que condensará definitivamente este principio. Para el autor el régimen de policía al que se aspira en esta época excede las tareas puramente disciplinares y represivas que llegarán a caracterizar la labor policial más adelante - se atiende también y por igual al bienestar de los individuos como recurso principal del Estado. De forma sintética, la policía de este período representaría el sistema de técnicas de gobierno que consideran «la actividad del hombre como elemento constitutivo de la fuerza del Estado» (Foucault 2008: 308); su finalidad, en consecuencia, es asegurar una reproducción social ordenada y funcional a los intereses del Estado (Foucault 2008: 313). Para tal fin se desplegaría una red de reglamentaciones que aspiran a controlar a los sujetos y sus actividades a lo largo de todo su periplo vital.

Sin embargo este proyecto simultáneo de subjetivación y assujettissement de sujeción de los individuos a una norma social y de producción de subjetividad (Foucault 1975) - evolucionará pronto hacia un modelo más abierto, más allá del archipiélago carcelario descrito de forma cruda en Surveiller et punir. Como Gramsci, Foucault sugiere que aparece en ese momento la idea de «sociedad civil» como una esfera independiente del Estado (Foucault 2008: 333), que no se entiende ya como una mera «serie de súbditos sometidos al soberano y a la intervención de la policía» (Foucault 2008: 335). La labor policial se diversifica y amplía, se incorpora la libertad individual como tecnología específica de gobierno de la población (Foucault 2008: 61) y se aíslan las tareas represivas en determinadas instituciones, adoptando la policía su forma contemporánea ${ }^{6}$. En ese período, entre los siglos XVIII y XIX, el gobierno reparte sus funciones en las labores de estudio y análisis de la población, regulación de los procesos que la conducen y control y castigo de los desórdenes puntuales que afloran en un régimen por lo demás estable. La gubernamentalidad encuentra entonces dos momentos interrelacionados: la gubernamentalidad policial, ocupada en conocer, controlar y, si es necesario, reprimir a la población; la gubernamentalidad liberal, ocupada en encauzar y regular, respetándola, la libertad de los individuos (Foucault 2008: 333-336). Como el propio autor destaca, se trata de dos momentos

6 Para una exploración de la vertiente específicamente urbana de esta innovación ver Joyce (2003).

EMPIRIA. Revista de Metodología de Ciencias Sociales. N. ${ }^{\circ}$ 27, enero-abril, 2014, pp. 49-72. ISSN: 1139-5737 DOI 10.5944/empiria.27.10862 
que pueden aparecer de forma simultánea, y no necesariamente sucesivos en el tiempo.

Recuperando los puntos señalados al hablar de Gramsci y proyectándolos sobre la noción de gubernamentalidad:

a. Es sobradamente conocida la caracterización foucaultiana del poder como un proceso que circula por la sociedad en su conjunto y que, por tanto, no se localiza exclusivamente en determinados centros de gobierno. Lo que ponen de relieve las reflexiones de los cursos en el Collège de France es que esa circulación se hace cada vez más centrífuga gracias a la nueva configuración del Estado como un ensamblaje de prácticas que conectan las instancias de gobierno con los niveles privado y cotidiano a través y en nombre de la libertad, el bienestar de la población y su defensa.

b. La labor pedagógica que Gramsci identificara en la función hegemónica es también un nodo en la constitución de la gubernamentalidad moderna. Aparece aquí, sin embargo, en formas más difusas y sutiles, como un momento más en el ejercicio de conducta de las conductas un ejercicio que presenta diversas formas a lo largo del tiempo pero que no mengua en la era moderna y tiene la vocación de abarcar el conjunto de la vida del sujeto.

c. Dicha labor pedagógica se despliega en una constelación de «prácticas del yo» en la que tiene un papel especialmente importante la movilización política de los «regímenes de veridición»: «los hombres gobiernan (a sí mismos y a los demás) mediante la producción de verdad» (Foucault 1991: 79). Esa producción de una verdad generalmente aceptada, de sentido común, modula también los hábitos y comportamientos de los sujetos: la gubernamentalidad se filtra al nivel de la vida cotidiana (Joyce 2003: 8) a través de la atención del gobierno a las actividades diarias de los individuos (Foucault 2008: 307) y se corporeiza en sus prácticas materiales.

d. Por último, como en Gramsci, la labor gubernamental aparece dividida en dos momentos fundamentales, el de anticipación al conflicto a través de la conducta de las conductas y el de represión del mismo a través del castigo a los comportamientos improcedentes. Se trata de funciones asimétricas: cuanto mayor sea la capacidad de anticipación al conflicto mejor se asegurará la estabilidad del gobierno (Foucault 2008: 307308). En consecuencia el ensamblaje de prácticas que constituye el Estado debe privilegiar aquellas destinadas a propiciar el autogobierno de los sujetos por interiorización de los principios de conducta y vigilancia mutua de los ciudadanos.

Pero, junto a estos puntos de contacto entre la hegemonía gramsciana y la gubernamentalidad foucaultiana, encontramos también diferencias sustanciales en ambas propuestas teóricas. De hecho son esas diferencias las que aconsejan la 
aproximación «herética» que estamos proponiendo; las respectivas aportaciones y vacíos conceptuales de estos dos autores se complementan y aseguran un análisis más integral del proceso político y su articulación con la vida cotidiana. En primer lugar Gramsci es, desde luego, el responsable de un refinado inicial de la lectura marxista de la dinámica de clases. Su visión compleja de las alianzas y conflictos inter- e intraclasistas — de la constitución de «bloques» sociales y el modo en que éstos sustentan una determinada hegemonía - supuso una sofisticación notable del discurso marxista tradicional. Después de Gramsci la ciencia política dispone de un marco de referencia más adecuado para leer la complejidad del juego político. Con todo, en ocasiones su análisis se apoya excesivamente en un cuadro conceptual —el del materialismo dialéctico coetáneo-que esencializa la noción de clase y la toma como una categoría ideal, no empírica. En una perspectiva diametralmente opuesta, el énfasis en la escritura de una historia sin sujeto - y, por tanto, sin clases- es una de las mayores debilidades de la propuesta foucaultiana, una y otra vez reprochada por los historiadores profesionales.

No es difícil, en todo caso, intuir la posibilidad de una vía intermedia entre ambas posturas, informada: (1) por la complejidad de la mirada gramsciana y su atención a los ensamblajes locales y temporales de poder - pero que prescinde de su faceta más mecanicista en el tratamiento de las clases sociales; y (2) por la advertencia foucaultiana de que es necesario comprender cómo funciona y circula el poder y no sólo quién lo detenta - pero prescindiendo de su terca ceguera ante la evidencia histórica de la existencia de proyectos de clase. En segundo lugar y con una repercusión metodológica aún mayor para nuestros intereses, puede buscarse una vía intermedia entre el análisis que cada autor hace de los mecanismos que realizan la hegemonía y la gubernamentalidad. Para Gramsci los instrumentos hegemónicos son fundamentalmente ideológicos y discursivos; aunque en ocasiones se refiere a la labor de cierto funcionariado y de los profesionales, sus notas no profundizan en la dinámica interna de dichas tareas. Foucault, por el contrario, defiende la necesidad de analizar pacientemente el modo en que los discursos y saberes se destilan en tecnologías de poder concretas. Con todo, tampoco él proporcionó una lectura sistemática del efecto que dichos conglomerados de técnicas tuvieron en las prácticas materiales de la población en general, más allá de sus conocidos monográficos sobre la clínica, el manicomio y la prisión. En definitiva, necesitamos nuevas aportaciones - aportaciones historiográficas - que desarrollen las perspectivas sugeridas por ambos autores para comprender: (1) cuál es la conexión entre ideología y prácticas materiales, cómo se filtra la hegemonía en la vida cotidiana y qué posibilidades de lectura de dicho proceso pueden ofrecer las ciencias sociales; y (2) cuáles son los mecanismos concretos que integran y coordinan determinadas técnicas para propiciar el cambio social, cómo debemos interpretar los efectos de estos mecanismos en el marco de un proyecto político más amplio.

En resumen, en sus momentos más extremos, tanto Gramsci como Foucault han propuesto lecturas de las relaciones de poder apoyadas en categorías y esquemas que resultan demasiado abstractos desde una perspectiva histórica. Por

EMPIRIA. Revista de Metodología de Ciencias Sociales. N. ${ }^{\circ}$ 27, enero-abril, 2014, pp. 49-72. ISSN: 1139-5737 DOI 10.5944/empiria.27.10862 
otra parte, ninguno de los dos llegó a cumplir por completo la promesa implícita en sus trabajos - el desvelamiento de las relaciones entre política y vida cotidiana a través del análisis de una serie de mediaciones discursivas y técnicas. Podemos enfrentarnos a buena parte de estas carencias si definimos un plano de inmanencia que haga posible acercar estos momentos teóricos. Estos defectos pueden ser superados con una investigación historiográfica que siga la siguiente pauta: (1) proporcionar el protagonismo necesario al análisis de las prácticas materiales, (2) emplear éstas como índice para comprender la formación de estructuras de experiencia y sentimiento, (3) apoyarse en dichas estructuras para estudiar los procesos de formación de subjetividad e identidad individual y colectiva, y (4) articular estos tres momentos en una lectura efectiva de la vida cotidiana, del lugar de ésta en los procesos de reproducción social y su papel en las dinámicas de cambio social.

Esta base metodológica debe sustentar una evaluación posterior del modo en que determinados grupos o bloques sociales regulan e intervienen en distintos puntos de esta arquitectura - el ensamblaje cotidiano de prácticas materiales, estructuras de experiencia y sentimiento, y procesos de formación de subjetividad e identidad - para asegurar una reproducción social afín a sus intereses y la consecución de sus proyectos de orden social, económico y político. El esfuerzo pionero de Henri Lefebvre por desarrollar una crítica de la vida cotidiana resulta clave para acompañar dicho movimiento y conectar el análisis de las condiciones materiales de existencia con las aportaciones de Gramsci y Foucault. Como ha señalado Stefan Kipfer (2008), Lefebvre describió la vida cotidiana como un conjunto de fenómenos contradictorios, atravesado por toda una serie de fuerzas sociales. Para él la vida cotidiana era un aspecto central en la reproducción de las relaciones sociales, incluyendo el trabajo pero también el ocio, el consumo, el lenguaje, etc. En un tono no muy lejano al empleado por Gramsci y, en cierto sentido, por Foucault, Lefebvre sugiere que la cristalización de la alienación y la ideología en las prácticas repetitivas de la rutina diaria es la mejor garantía contra la revolución, al implantar un sentido de lo evidente e inevitable en la experiencia del ser humano. En consecuencia lo cotidiano es un asiento del poder, «el suelo mismo sobre el que se levanta la gran arquitectura de la política» (Lefebvre 1976: 89).

Teniendo en cuenta esta necesidad de atención a la vida cotidiana y los parámetros metodológicos para guiar un estudio histórico concreto de su posición en el proceso de reproducción social, podemos integrar las perspectivas de la hegemonía y la gubernamentalidad como momentos complementarios en la lectura de las estructuras de poder. Articulando los aspectos anteriores con los puntos de contacto entre Gramsci y Foucault que habíamos identificado, tendremos la siguiente pauta de investigación:

a. Análisis de la circulación del poder más allá de las instituciones estatales, atendiendo al modo en que los proyectos políticos integran la sociedad civil en su estrategia de orden social, 
b. Identificación de prácticas de gobierno dirigidas a la formación de subjetividades a través de una constelación de momentos pedagógicos,

c. Estudio de la filtración e interiorización del proyecto de gobierno en el nivel de la vida cotidiana y las prácticas materiales,

d. Comprensión del conjunto de procesos como una estrategia ideada para mitigar el conflicto abierto y gobernar a través del consenso y la estabilidad social.

Estos son, en suma, los elementos metodológicos de sustento para una historia social capaz de conectar de forma efectiva lo macro y lo micro, el nivel de la política convencional y el de la vida cotidiana, los proyectos de clase y los procesos de reproducción de las relaciones sociales, etc. Pero ¿dónde queda el espacio en este esquema? ¿De qué forma conecta este análisis histórico de los procesos sociales y su mediación política con los aspectos espaciales y urbanísticos?

\section{EL ESPACIO Y EL TERRITORIO EN LAS COORDENADAS DEL PROYECTO HEGEMÓNICO-GUBERNAMENTAL}

En las décadas recientes han sido numerosos los esfuerzos por proporcionar al espacio un papel en el discurso de las ciencias sociales, especialmente por parte de los geógrafos (ver, por ejemplo, Massey \& Allen 1984; Gregory \& Urry 1985; Soja 1989), pero también en disciplinas como la sociología, la antropología y los estudios culturales (por ejemplo Lipietz 1979; Castells 1986; Gottdiener 1985; Jameson 1991; Giddens 1995; Haraway 1996), la filosofía y la historia de las ideas (Rabinow 1989; Casey 1997; Elden 2001), etc. En contrapartida algunos urbanistas han atendido especialmente a la dimensión social de su labor en contribuciones quizá más limitadas pero igualmente productivas (por ejemplo Brenner et al, 2012; Davis 2001; 2006; Fainstein 2010; Friedman 2002; 2011; ver especialmente las respuestas proporcionadas por un selecto grupo de urbanistas internacionales en Sevilla-Buitrago, 2013). Este debate es de sumo interés para el proyecto de investigación que estoy esbozando. Como se apresurarían a advertir éstos y otros autores, el espacio es un factor clave en la mediación y dirección política de los procesos de reproducción social, especialmente en el nivel de la vida cotidiana: la espacialización de las contradicciones sociales y su tratamiento a través de mecanismos específicos de gobierno contribuye a naturalizarlas, integrándolas así de forma inadvertida en la cotidianidad y disolviendo su potencial carácter conflictivo.

Aunque no agotan esta faceta, los estudios urbanos desarrollados en Francia entre finales de la década de 1960 y la de 1980 suponen una aportación aún relevante en la comprensión de esa articulación entre espacio, reproducción social y vida cotidiana. Además del trabajo de Henri Lefebvre y para mencionar sólo algunas de las más conspicuas, cabe recordar las contribuciones de Alain Lipietz

EMPIRIA. Revista de Metodología de Ciencias Sociales. N. ${ }^{\circ}$ 27, enero-abril, 2014, pp. 49-72. ISSN: 1139-5737 DOI 10.5944/empiria.27.10862 
— quién señaló que el espacio «no es otra cosa que la reproducción social en su dimensión más material» (Lipietz 1979: 9)—o, en una línea muy distinta, de Michel de Certeau, que desarrolló una inspiradora lectura de las prácticas espaciales cotidianas como momentos de micro-resistencia urbana (De Certeau 2000). Es también conocida la atención que Manuel Castells prestó a la ciudad como espacio de reproducción de la fuerza de trabajo en La cuestión urbana. Castells - en un gesto teórico quizás demasiado grueso- equiparaba «vida cotidiana» y «reproducción social», y presentaba «lo urbano» no como una forma espacial, sino como expresión de la organización del proceso de reproducción social (Castells 1986: 474,488):

El espacio urbano se convierte así en el espacio definido por una cierta porción de la fuerza de trabajo, delimitada, a un tiempo, por un mercado de empleo y por una unidad (relativa) de su existencia cotidiana ... «Lo urbano» [connota] el proceso de reproducción de la fuerza de trabajo, y el «espacio urbano» [expresa] las unidades articuladas de [tal] proceso. (Castells 1986: 279)

Para Castells (1986: 280-281, 489) la ciudad se convierte bajo el capitalismo en un espacio estructurado en primer lugar por los procesos de consumo colectivo que aseguran el mantenimiento de la clase obrera: «las ciudades son lugares para la provisión de servicios que socializan el proceso de reproducción de la fuerza de trabajo, minimizando para el capital los costes de transporte, asistencia sanitaria, educación y alojamiento, servicios necesarios que son total o parcialmente financiados y organizados por el Estado» (Warde 1985: 205).

Pero, a pesar de su relevancia, la excesiva abstracción de buena parte de estas contribuciones y una cierta fijación en la escala urbana plantean límites a diversos niveles del análisis. El caso de Castells es revelador en este sentido. Como él mismo señala en varios puntos (Castells 1986: 290, 475), su marxismo estructuralista no puede proporcionar una explicación satisfactoria de casos concretos, cuya particularidad siempre excede localmente los intentos de abstracción teórica y requiere un análisis históricamente específico, atento al modo en que una determinada trayectoria evolutiva determina el resultado final. Otra limitación, señalada con frecuencia, es la fetichización teórica de la ciudad. Aunque Castells considera el concepto de «ciudad» una mera vulgarización del más «científico» «unidad urbana» ${ }^{7}$, lo cierto es que su trabajo conserva la ciudad como objeto de estudio, infravalorando las interacciones con su contexto y el proceso de co-determinación y evolución mutua.

También en el marco más amplio del «giro espacial» que mencionábamos se ha advertido de forma insistente que «lo espacial» no puede ser asumido acríticamente. El «espacio», como la «ciudad», requiere una conceptualización relacional y evolutiva capaz de presentar todas las aristas del fenómeno (Massey 2005:

7 Para Castells la «unidad urbana» constituye una especie de ámbito espacialmente diferenciable de reproducción de la fuerza de trabajo: «La unidad urbana es al proceso de reproducción lo que la empresa es al proceso de producción: unidad específica articulada con las otras unidades que forman el conjunto del proceso» (Castells 1986:474).

EMPIRIA. Revista de Metodología de Ciencias Sociales. N. ${ }^{\circ}$ 27, enero-abril, 2014, pp. 49-72. ISSN: 1139-5737 DOI 10.5944/empiria.27.10862 
9; Harvey 2009: 133-165). De hecho, la propia tradición marxista ha presentado en otras ocasiones una sensibilidad especial hacia esta naturaleza compleja de las formaciones socioespaciales. Para mencionar sólo los hitos que considero más relevantes, pensemos en la atención marxiana a las relaciones campo-ciudad y su desarrollo histórico; el estudio de las relaciones centro-periferia-entorno no capitalista y su papel en la expansión imperialista en los análisis de Lenin y Rosa Luxemburg; la agudeza de Henri Lefebvre para comprender el cambio urbano en una red más amplia de relaciones de mundialización; la apuesta de Doreen Massey por desarrollar una concepción global del lugar como fundamento de un localismo internacionalista; la conceptualización de las políticas de la escala como momento clave en la división social del espacio y la necesidad de leer crítica y transversalmente dicha segmentación escalar en el trabajo de Erik Swyngedouw y Neil Smith; o la más reciente llamada de Neil Brenner a abandonar las categorías tradicionales de campo y ciudad para redescubrir el proceso de urbanización como verdadero desencadenante del cambio socioespacial. Todas estas aportaciones redundan, de un modo u otro, en una comprensión del espacio a partir de las prácticas que lo entretejen y articulan socialmente, en una atención a las prácticas espaciales o espacialidades de agencias e individuos. En otros términos, son los aspectos procesuales y relacionales los que reciben la carga ontológica de la producción del espacio, no una batería más o menos exhaustiva de categorías estáticas.

En cualquier caso «espacio» y «ciudad» son términos escurridizos y contestados en la teoría reciente. En su lugar sugeriría los conceptos de «territorio» y «territorialidad» como herramientas de análisis más precisas para una historia social de la planificación que asimile esta concepción relacional de lo espacial. Con respecto al concepto de «espacio», el de «territorio» presenta la ventaja de su materialidad, su referencia a enclaves concretos y acotados y por tanto libres de la confusión implícita en una concepción abstracta del espacio como categoría absoluta; con respecto al concepto de «ciudad» el «territorio» nos permite integrar en el análisis la relacionalidad constitutiva del espacio al presentar realidades materiales entretejidas a través de múltiples escalas, del cuerpo al mundo. Se trata, en todo caso, de una categoría objeto ella misma de una agitada discusión en las últimas décadas; me apresuraré por tanto a aclarar que persigo una caracterización muy específica del concepto de «territorio» y el complementario de «territorialidad» - una caracterización que los hace inmediatamente operativos para nuestros intereses. A pesar de que ambas son utilizadas comúnmente sin mayores pretensiones intelectuales ${ }^{8}$, encontramos desarrollos recientes que matizan estas categorías, haciéndolas más productivas tanto desde una perspectiva teórica como con vistas a su instrumentalización empírica. Así, por ejemplo, Anssi Paasi (2003) o Stuart Elden (2010) han propuesto la necesidad de estudiar el territorio como un proceso o una práctica, un constructo social sostenido en

8 Especialmente en la teoría política para referirse al espacio sobre el que se ejerce jurisdicción o soberanía y en la práctica de la planificación y las políticas espaciales para referirse a ámbitos de administración supramunicipal.

EMPIRIA. Revista de Metodología de Ciencias Sociales. N. ${ }^{\circ}$ 27, enero-abril, 2014, pp. 49-72. ISSN: 1139-5737 DOI 10.5944/empiria.27.10862 
el tiempo por un conjunto de agencias y mediaciones institucionales, discursivas y materiales. Con todo, en estas aproximaciones el concepto de territorialidad aparece como una mera derivada del territorio, el ejercicio que persigue la conservación de la autoridad política sobre una determinada unidad espacial.

En mi opinión, un análisis que busque comprender la incardinación de los fenómenos espaciales en la vida cotidiana y los procesos de reproducción social encontrará más oportuno seguir el camino contrario: entender el territorio como el resultado de un determinado ensamblaje de prácticas espaciales que denominaremos territorialidad. Es éste el camino seguido por los que podemos considerar autores de referencia en la teorización del concepto: Claude Raffestin y Robert D. Sack. A pesar de las distancias, ambos coinciden en la interpretación de la territorialidad como un modo de ejercicio de poder sobre terceros por medios indirectos, en este caso espaciales. Como el lector adivinará, esta línea de lectura resuena totalmente con lo expuesto en el apartado anterior al presentar los conceptos de «hegemonía»y «gubernamentalidad». A pesar de que los trabajos de Sack $(1983 ; 1986)$ son mucho más conocidos por su extensa difusión en el mundo anglosajón, es quizá la aportación del geógrafo suizo Claude Raffestin la que resulta más cercana a nuestra exposición - de hecho su principal aportación, el volumen Pour une geographie du pouvoir (Raffestin 1980) se presenta en su momento como una traslación espacial del trabajo coetáneo de Michel Foucault, incorporando además fuertes influencias de Henri Lefebvre.

Para Raffestin el territorio es espacio codificado, un determinado ámbito espacial estructurado por una serie de códigos o sistemas de regulación social que persiguen determinados objetivos: «El territorio es generado a partir del espacio, es el resultado de la acción de un actor sintagmático ... Al ocupar ... un espacio, el actor [lo] territorializa» (Raffestin 1980: 138, 143, 129). El territorio es «una producción ... inscrita en un campo de poder», sostenida por un conjunto de mediadores - prácticas y conocimientos, técnicas y ciencias- condicionados por la infraestructura económica y embebidos en una serie de códigos, un sistema de regulación de significados, de accesos y exclusiones (Raffestin 1980: 130; 1997: 100). El territorio «no es un objeto», sino «un proceso en evolución perpetua»; no es sólo un producto, sino también un medio de producción. No es, en suma, sólo un fin en sí mismo - una reserva de recursos o activos con la cual los actores sociales mantienen una relación finalista - sino también el instrumento que dichos actores emplean para controlar y someter a otros individuos: el punto de triangulación gracias al cual un grupo hegemónico media su relación con las clases subalternas (Raffestin 1982: 168; 1980: 143-144). En todo caso Raffestin advierte que la implementación específica de estas estrategias depende de las condiciones sociales e históricas en las que aparecen y son puestas en práctica, de la evolución de las técnicas y los conocimientos que las despliegan; en suma, de las territorialidades históricas que movilizan dichos mecanismos para alcanzar un determinado objetivo.

Raffestin define «territorialidad» como «el sistema de relaciones que el hombre (sic), como miembro de una colectividad, mantiene con la exterioridad y la alteridad con la ayuda de mediadores con el fin de garantizar su autonomía»

EMPIRIA. Revista de Metodología de Ciencias Sociales. N. ${ }^{\circ}$ 27, enero-abril, 2014, pp. 49-72. ISSN: 1139-5737 DOI 10.5944/empiria.27.10862 
(Raffestin 1988: 48-50). Esta concepción «invierte el orden convencional de la geografía, porque el punto de partida no es ya el espacio, sino los instrumentos y códigos de los actores que dejan sus huellas y signos en el territorio» (Raffestin 1986: 94). Sack presenta un cuadro conceptual parcialmente similar. Él define «territorialidad» como «una estrategia espacial para afectar, influenciar o controlar los recursos y la población a través del control de un área determinada», que este autor denomina «territorio» (Sack 1986: 1-2,19); se trata de una estrategia dirigida a regular un sistema de interacción y acceso diferencial a las cosas y las personas (Sack 1983: 55). Sin embargo, a pesar de la cercanía de algunas interpretaciones, la propuesta de Raffestin parece más oportuna para nuestros intereses porque (1) pone especial énfasis en las técnicas que sostienen la territorialidad y en la dialéctica histórica entre conocimiento y prácticas, lo que nos mantiene atentos a las modalidades de producción del territorio; (2) para Sack el territorio es simplemente el área de influencia sobre la cual se ejerce la territorialidad, mientras que para Raffestin se trata de un ensamblaje material con un significado social mucho más rico y que, en última instancia, está sometido a una dinámica de producción / reproducción permanente. Como él mismo sugiere, «todo territorio está inmerso en un proceso de territorialización - desterritorialización - reterritorialización» (Raffestin 1988: 49), es decir, en una dinámica de codificación del espacio, de posterior erosión (decodificación) y sustitución (recodificación) de los sistemas de relaciones que lo constituyen. Tenemos aquí, por tanto, una caja de herramientas analíticas idónea para comprender la evolución de esos conglomerados socioespaciales que son los territorios y para entender el papel que los proyectos políticos y las técnicas - la planificación entre ellastienen en ese devenir histórico.

Como mencionaba antes, la territorialidad se entiende así como una modalidad de ejercicio de poder, una estrategia en la que un sujeto o grupo social traza su proyecto de influencia y dominio sobre otros grupos de población por medios indirectos, a través de una mediación que instrumentaliza el espacio para dichos fines. La posibilidad de conexión con el marco de análisis implícito en los conceptos de hegemonía y gubernamentalidad es obvia. Como hemos visto, ambas nociones identifican un desvío en las prácticas de gobierno que evita presentar el dominio político en la forma descarnada de un control directo de las poblaciones, empleando para ello una constelación de prácticas, técnicas y discursos dirigidos a conducir los comportamientos y formar subjetividades, asegurando de ese modo la aquiescencia de la población con el orden social que pretende implantarse. La territorialidad sería en este marco un momento - el momento espacial o territorial — de ese proyecto hegemónico / gubernamental más amplio. Podríamos hablar así de:

- Hegemonía territorial, que definiremos como la práctica de diseminación de formas de uso y concepción del espacio — de códigos que territorializan ese espacio- compatibles con el proyecto de orden socioespacial del bloque dominante. 
- Gubernamentalidad territorial, que definiremos como la racionalidad de gobierno que, apoyándose en un conjunto de técnicas y saberes orgánicos, implementan esos usos y concepciones, bien desde una perspectiva policial (conocer, controlar y disciplinar las territorialidades de la población) o liberal (propiciar, favorecer e incentivar ciertas territorialidades).

Pero si la hegemonía y la gubernamentalidad pueden declinarse territorialmente, si el proyecto de orden social que persiguen despliega una territorialidad propia, ello no significa que dicha territorialidad agote el campo de los territorios posibles, de las codificaciones posibles del espacio. Lo espacial se caracteriza por su apertura constitutiva, por ser la condición ontológica de una multiplicidad de presencias y trayectorias sociales que coexisten y abren el futuro a horizontes (políticos) alternativos (Massey 2005: 9). La territorialidad del bloque dominante - esa territorialidad que se integra en un proyecto hegemónico o gubernamental determinado- podrá aspirar, a su vez, a convertirse en una territorialidad hegemónica, pero para prevalecer ha de coexistir y luchar para prevalecer con otras territorialidades - territorialidades subalternas - otras codificaciones del espacio, otras prácticas, otras formas de asignación de usos, discursos e imaginarios sobre el espacio. En definitiva, podemos hablar de una lucha de territorialidades (Sevilla Buitrago 2008) en la medida en que los grupos sociales luchan para imponer, de forma consciente o inconsciente, su propia ordenación del espacio.

\section{LA PLANIFICACIÓN COMO TÉCNICA EN LA LUCHA DE TERRITORIALIDADES}

Podemos ahora anticipar el papel histórico que la planificación ha tenido en este proceso de lucha de territorialidades y en su articulación con los proyectos más amplios de regulación de la reproducción social desde los albores del capitalismo9. Toda territorialidad se sustenta en un conjunto de técnicas y saberes, más o menos rudimentarios o sofisticados. La planificación se ha presentado históricamente en esa forma: como una tecnología, una racionalidad que coordina un conjunto de saberes y técnicas dirigidos al disciplinamiento, propiciación e incentivación de determinadas prácticas espaciales (Boyer 1997: 59-63). En este sentido la planificación se inscribe en el proceso histórico de producción del territorio - en la constante codificación-decodificación-recodificación del espacio- como elemento de una lógica de gobierno más amplia, actuando en función de condiciones sociales, espaciales e históricas localmente específicas y variables en el tiempo. No se trata sólo de una técnica al servicio del poder. Esa lectura es demasiado cruda, tanto si se presenta en la forma de un supuesto

9 La historiografía convencional presenta la planificación como una disciplina que aparece para corregir las contradicciones de la ciudad industrial a partir de la segunda mitad del siglo XIX y, por tanto, fruto de una dinámica capitalista ya madura. Por el contrario, una concepción más amplia del rol social de la planificación exige adelantar su origen a la fase de acumulación primitiva, en plena transición del feudalismo al capitalismo (Sevilla Buitrago 2010; Sevilla-Buitrago 2012).

EMPIRIA. Revista de Metodología de Ciencias Sociales. N. ${ }^{\circ}$ 27, enero-abril, 2014, pp. 49-72. ISSN: 1139-5737 DOI 10.5944/empiria.27.10862 
lado oscuro de la planificación (Yiftachel 1998), como intervención de la «clase dominante» en la formación social para asegurar la reproducción ampliada de la fuerza de trabajo (Castells 1986: 475), como proyecto modernista de normalización de minorías sociales (Sandercock 1998), etc.

La planificación constituye, más bien, el soporte técnico-científico de una territorialidad inscrita en un proyecto hegemónico/gubernamental más profundo. Representa un momento más - el momento espacial o territorial_ en la construcción de aparatos de mediación para la regulación y conducta de la vida cotidiana de la población y, especialmente, de las clases subalternas. Es fundamental partir de la atención a la particularidad histórica y geográfica de cada uno de los posibles escenarios de análisis: en cada momento, en cada lugar, las alianzas y antagonismos sociales, la configuración de fuerzas productivas y relaciones de producción, su articulación con las instituciones y la cultura de la época, las espacialidades y su incardinación en las prácticas materiales más amplias, los conglomerados territoriales preexistentes, etc. son localmente específicos y dependen de su trayectoria previa. Cuando se trata de la producción de territorios, no es posible trazar esquemas universales que asignen estadísticamente roles precisos en el proceso a determinadas clases o fracciones de clase, que adscriban técnicas, discursos o modelos concretos a determinadas situaciones, que identifiquen relaciones causa-efecto necesarias frente a ciertos entramados territoriales, etc. Sólo es posible identificar una lógica procesual, una constante práctica de territorialización en la cual los grupos dominantes intentan asegurar una reproducción del territorio afín a sus intereses, atrayendo por vías diversas a sus antagonistas sociales hacia una nueva territorialidad, una nueva cotidianidad espacial objetivamente contradictoria con sus intereses - o intentando eliminar por métodos más expeditivos las codificaciones subalternas si lo anterior no es posible.

Podemos hablar por tanto de una lógica de desposesión de capitales territoriales: los grupos sociales hegemónicos o con aspiraciones a serlo intentarán erosionar paulatinamente la capacidad de territorialización de los grupos antagonistas, su facultad para apropiarse y organizar el espacio a través de prácticas cotidianas. ¿Por qué este énfasis en los patrones de vida cotidiana de los grupos subalternos? Conformados según relaciones, tiempos y espacios específicos de formas económicas en vías de extinción, estos patrones - estos vestigios de un entorno no capitalista - han obstaculizado espontáneamente el avance y consolidación histórica del capitalismo por el mero hecho de arrastrar inercias propias de modos de vida, socialización y territorialización pretéritos, relativamente ajenos a la revolución permanente en la esfera productiva y apoyados en una realización de las necesidades de reproducción independiente del mercado (Dodgshon 1998). Esta asincronía entre la vanguardia de la economía formal y los modos generales de vida ha hecho necesaria la implementación de instrumentos para la intervención y regulación de esos procesos, lo que Göran Therborn denominó «mecanismos de reproducción social» (Therborn 1979). Dichos mecanismos han corregido o suprimido los patrones de reproducción social contrarios al sistema y se han encargado de habilitar los marcos para la mate- 
rialización de otros nuevos, completamente funcionales al mismo. En el caso de los mecanismos espaciales de reproducción social, esa labor no comprende sólo la eliminación de prácticas conflictivas y su sustitución por otras nuevas; dicha tarea ha de llevarse a cabo en el seno de territorios ajenos al nuevo orden, que sobredeterminan la concepción y aplicación de aquellos mecanismos.

En todo caso, la lógica de desposesión que hemos mencionado atraviesa momentos muy diversos y no necesariamente lineales, sino pautados por impulsos discretos y desiguales de innovación y estancamiento. Es preciso, por tanto, rastrear las territorialidades históricas que moviliza cada práctica hegemónica para comprender estos momentos de desposesión, de supresión de la capacidad de territorializar de los grupos subalternos, para entender cómo la nueva territorialidad anida en un territorio extraño y lo desterritorializa / reterritorializa paulatinamente, imponiendo un nuevo orden socioespacial. El proceso de desposesión puede ir de la mera y simple desposesión de recursos materiales (derechos de acceso a y/o apropiación de la tierra y otros recursos, etc.), a la eliminación de capitales sociales (comportamientos e instituciones espaciales, formas de uso y organización colectiva del espacio, etc.), a la suplantación de representaciones espaciales (imaginarios urbanos, memorias colectivas, paisajes e identidades, etc.). En suma, los grupos subalternos se ven sometidos a una revolución permanente de los marcos de referencia de su espacio-tiempo social y son paulatinamente privados de sus capacidades de organización y experiencia autónomas, de su capacidad de autogestión del espacio de vida cotidiana a medida que sus prácticas espaciales son reescritas, recodificadas, reterritorializadas de forma heterónoma. Por su parte y a nivel técnico, el discurso y práctica de la planificación se desarrolla según una genealogía propia de racionalidades espaciales (Huxley 2006), desde el carácter burdo de las primeras políticas de reestructuración y consolidación de la tierra, a la mentalidad determinista de los primeros reformistas urbanos, a concepciones mucho más sofisticadas y conscientes de la necesaria articulación entre los distintos mecanismos de reproducción social para la consecución de los objetivos marcados por los bloques hegemónicos.

Por tanto la planificación espacial aparece como una emergencia sistémica en la evolución histórica de las formas de gobierno, uno de los elementos institucionales de los proyectos hegemónicos / gubernamentales que, en los albores del capitalismo, sustituyen paulatinamente los viejos modos de organización del poder basados en el dominio directo y coercitivo. Codificar el espacio de acuerdo a los intereses de ese proyecto hegemónico, teniendo en cuenta la configuración socioespacial de los territorios prexistentes y con la ayuda de nuevas técnicas regladas, reproducibles y respaldadas por la administración pública; articular estratégicamente códigos espaciotemporales y códigos de vida cotidiana para propiciar prácticas espaciales aceptables, regulando el conflicto social y participando así en la formación del nuevo orden: éstas son las tareas que propician la aparición de la planificación, acompañando al capitalismo en su proceso de gestación y en su transición a cada una de sus fases subsiguientes. La planificación se convierte así y a través de formas variables en el dispositivo responsable de transformar el territorio heredado por cada nuevo bloque hegemónico, eliminan-

EMPIRIA. Revista de Metodología de Ciencias Sociales. N. ${ }^{\circ}$ 27, enero-abril, 2014, pp. 49-72. ISSN: 1139-5737 DOI 10.5944/empiria.27.10862 
do los soportes espacio-temporales de las viejas prácticas sociales que contradicen los intereses del orden en curso de formación.

\section{CONCLUSIONES}

Es a través de este esquema de investigación que podemos aspirar a desarrollar una historia efectiva del gobierno de los procesos de reproducción social a través de técnicas específicas de regulación espacial de la vida cotidiana. Los historiadores del urbanismo se han demorado buscando una «constitución interna» para la disciplina, leyendo las técnicas, reuniendo elementos cuya adecuada combinación habría precipitado en un determinado momento en una nueva lógica reguladora, que podríamos denominar «planificación». Por supuesto no hay acuerdo sobre qué técnica, combinada con qué saberes y en qué momento y lugar, produce la alquimia. Sospecho que se trata de una indagación vana. El cometido que la historiografía debería fijarse es, por el contrario, el de desvelar la «constitución externa» de la planificación, su nacer a la llamada de determinadas hegemonías sociales históricas. Desde esta perspectiva, la planificación surge cuando las formas de poder han alcanzado un grado de madurez suficiente y comienzan a articular un proyecto de hegemonía social que interpela a las técnicas de ordenación del territorio. Materializar en el espacio ese proyecto, producir una nueva configuración territorial que facilite otras instancias de gobierno y ejercicio del poder, participando así en la formación del nuevo orden: esta es la tarea con la cual y a la cual nace la planificación, vecina a la aparición del capitalismo.

Una historiografía renovada que lea el pasado en este sentido puede contribuir sustancialmente en el ejercicio de deconstrucción teórico-práctica de las técnicas de ordenación del espacio, trazando la historia de su presente, del modo en que han llegado a integrarse en nuestras actuales estrategias de planificación urbana y territorial. Se trata, en suma, de leer a contrapelo la historia de nuestras técnicas para identificar los aspectos desposeedores que anidan en ellas, intentar suprimirlos e invertir la situación, transformándolas o descartándolas por otras nuevas. Comprender, por ejemplo, que fue necesario suprimir tierras comunales para crear parques públicos y que en ese paso se perdieron capitales de gestión colectiva del espacio; que las redes de equipamientos públicos surgieron como un mecanismo para la penetración del proyecto hegemónico del que hemos hablado; que las políticas residenciales y las mejoras que conllevaban fueron planteadas inicialmente en muchos lugares como recompensa por la adopción de ciertos patrones de conducta, socialización y compromiso con el trabajo asalariado; que los asentamientos-modelo y las políticas de la comunidad de desplegaban fueron concebidos como articulación de las soluciones anteriores en el marco de un nuevo esquema de welfare condicionado a perfiles específicos de clase, género, raza y etnia. Desde luego no se trata de desechar, sin más, un instrumental construido lentamente a lo largo de décadas. La lectura crítica que he esbozado en este artículo no reclama, por ejemplo, que debamos prescindir de 
la zonificación, de los parques públicos o los equipamientos que nuestras leyes exigen. Pero, sin duda, una reflexión más profunda sobre su cometido inicial y el modo en que estas soluciones han llegado a hacerse «naturales» en la normativa y la metodología de la planificación nos apartaría del callejón sin salida en que buena parte de las técnicas urbanísticas parece encontrarse en la actualidad en lo que se refiere a la producción de territorios socialmente más justos y que permitan la realización colectiva del derecho a la ciudad.

Aunque es difícil sostener este argumento frente a los propios interesados - especialmente representantes políticos, planificadores y urbanistas implicados en la cobertura ideológica de la producción institucional del espacio- es necesario adoptar esta perspectiva si queremos comprometer los discursos y técnicas urbanísticas en un proyecto de equidad y justicia social. Confío en que la historia social de la planificación que aquí he esbozado será capaz de ayudar en ese empeño aportando una revisión crítica exhaustiva y sin concesiones corporativas, que nos permita desvelar las deudas históricas de esta disciplina y de otorgarle una nueva legitimidad fundada en el compromiso sin fisuras con un proyecto de recuperación de lo común bajo coordenadas radicalmente democráticas.

\section{BIBLIOGRAFÍA}

ALTHUSSER, L. (1988): Ideología y aparatos ideológicos del estado: Freud y Lacan, Buenos Aires, Nueva Visión.

BARNES, T. (2012): «Notes from the Underground: Why the History of Economic Geography Matters: The Case of Central Place Theory», Economic Geography, 88, pp. 1-26.

BATES, T. R. (1975): «Gramsci and the Theory of Hegemony», Journal of the History of Ideas, 36, pp. 351-366.

BOYER, C. (1997): Dreaming the Rational City. The Myth of American City Planning, Cambridge (Mass.), The MIT Press.

BRENNER, N., MARCUSE, P., \& MAYER, M. (2012) Cities for People, Not for Profit. Critical Urban Theory and the Right to the City, New York, Routledge.

BURCHELL, G., GORDON, C., \& MILLER, P. (eds.) (1991): The Foucault Effect. Studies in Governmentality, Chicago, University of Chicago Press.

CASEY, E. (1997): The Fate of Place. A Philosophical History, Berkeley, University of California Press.

CASTELLS, M. (1986) La cuestión urbana, Madrid, Siglo XXI.

DAVIS, M. (2001): Magical Urbanism. Latinos Reinvent the U.S. City, New York, Verso.

DAVIS, M. (2006): Planet of Slums, New York, Verso.

DE CERTEAU, M. (2000): La invención de lo cotidiano I. Artes de hacer, México D.F., Universidad Iberoamericana.

DEAN, M. (1994): Critical and effective histories. Foucault»s methods and historical sociology, London, Routledge.

DEAN, M. (1999): Governmentality - Power and Rule in Modern Society, London, Sage, 1999.

DIKEÇ, M. (2012): «Space as a mode of political thinking», Geoforum, 43, pp. 669-676.

DODGSHON, R. (1998): Society in time and space: a geographical perspective on change, Cambridge, Cambridge University Press.

EMPIRIA. Revista de Metodología de Ciencias Sociales. N. ${ }^{\circ}$ 27, enero-abril, 2014, pp. 49-72. ISSN: 1139-5737 DOI 10.5944/empiria.27.10862 
DRIVER, F. (1985): «Power, space, and the body: a critical assessment of Foucault»S Discipline and Punish», Environment and Planning D: Society and Space, 3, pp. 425-446.

ELDEN, S. (2001): Mapping the Present: Heidegger, Foucault and the Project of a Spatial History, London, Continuum.

ELDEN, S. (2010): «Land, terrain, territory», Progress in Human Geography, 34, pp. 799-817.

ELDEN, S. (2007): «Governmentality, calculation, territory», Environment and Planning D: Society and Space, 25, pp. 562-580.

FAINSTEIN, S. (2010): The Just City, Ithaca, Cornell University Press.

FEMIA, J. V. (1979): «The Gramsci phenomenon: some reflections», Political Studies, 27, pp. 472-483.

FOUCAULT, M. (1975): Surveiller et punir. Naissance de la prison, Paris, Gallimard.

FOUCAULT, M. (1991): «Questions of Method», en Burchell, Graham, Gordon, Colin \& Miller, Peter (eds.) The Foucault Effect. Studies in Governmentality, Chicago, University of Chicago Press, pp. 73-86.

FOUCAULT, M. (2008): Seguridad, territorio, población, Madrid, Akal.

FOUCAULT, M. (2009): Nacimiento de la biopolítica, Madrid, Akal.

FRIEDMANN, J. (2002): The Prospect of Cities, Minneapolis, University of Minnesota Press.

FRIEDMANN, J. (2011): Insurgencies. Essays in Planning Theory, New York, Routledge.

GIDDENS, A. (1995): A contemporary critique of historical materialism, Stanford (California), Stanford University Press.

GOTTDIENER, M. (1985): The Social Production of Urban Space, Austin, University of Texas Press.

GRAMSCI, A. (2007): Quaderni del carcere. Edizione critica dell»Istituto Gramsci, Torino, Einaudi.

GREGORY, D. \& URRY, J. (eds.) (1985): Social Relations and Spatial Structures, London, Macmillan.

GUNN, S. (2006): «From Hegemony to Governmentality: Changing Conceptions of Power in Social History», Journal of Social History, 39, pp. 705-720.

GUNN, S. (2011): Historia y teoría cultural, Valencia, Universitat de València.

HARAWAY, D. (1996): «Situated Knowledges: The Science Question in Feminism and the Privilege of Partial Perspective», en Agnew, J., Livingstone, D., Rogers, A., (eds.) Human Geography: An Essential Anthology, Oxford, Blackwell, pp. 108-128.

HARVEY, D. (2009): Cosmopolitanism and the Geographies of Freedom, New York, Columbia University Press.

HUXLEY, M. (2006): «Spatial rationalities: order, environment, evolution and government», Social \& Cultural Geography, 7, pp. 771-787.

JAMESON, F. (1991): Postmodernism, or, The cultural logic of late capitalism, Durham, N.C., Duke University Press.

JESSOP, B. (2001): «From micro-powers to governmentality: Foucault»s work on statehood, state formation, statecraft and state power», Political Geography, 26, pp. 34-40.

JOYCE, P. (2003): The Rule of Freedom: Liberalism and the Modern City, London, Verso.

KIPFER, S. (2008): «How Lefebvre urbanized Gramsci: hegemony, everyday life, and difference», en GOONEWARDENA, K., KIPFER, S., MILGROM, R., \& SCHMID, C. (eds.) Space, Difference, Everyday Life. Reading Henri Lefebvre, New York, Routledge, pp. 193-211. 
LACLAU, E. \& MOUFFE, C. (1987): Hegemonía y estrategia socialista. Hacia una radicalización de la democracia, Madrid, Siglo XXI.

LEFEBVRE, H. (1976): The Survival of Capitalism: Reproduction of the Relations of Production, London: Allison \& Busby.

LIPIETZ, A. (1979): El capital y su espacio, México, D.F., Siglo XXI.

MACDONALD, B.J. (2002): «Marx, Foucault, genealogy», Polity, 34, pp. 259-284.

MASSEY, D. (2005): For Space, London, Sage.

MASSEY, D. \& ALLEN, J. (eds.) (1984): Geography Matters! A Reader, Cambridge, Cambridge University Press.

PAASI, A. (2003): «Territory», en John Agnew, Katherine Mitchell \& Gerard Toal (eds.) A Companion to Political Geography, Oxford, Blackwell.

PICKERING, A. (1995): The mangle of practice: Time, agency and science, Chicago, University of Chicago Press.

RABINOW, P. (1989): French Modern. Norms and Forms of the Social Environment, Cambridge (Mass.), The MIT Press.

RAFFESTIN, C. (1980): Pour une géographie du pouvoir, Paris, Litec.

RAFFESTIN, C. (1982): «Remarques sur les notions d»espace, de territoire et de territorialité», Espaces et sociétés, 41, pp. 167-171.

RAFFESTIN, C. (1986): «Territorialité: concept ou paradigme de la géographie sociale?», Geographica Helvetica, 2, pp. 91-96.

RAFFESTIN, C. (1988): «Le territoire, la territorialité et la nuit», Actualités psychiatriques, 2 pp. 48-50.

RAFFESTIN, C. (1997): «Le rôle des sciences et des techniques dans les processus de territorialisation», Cahiers Vilfredo Pareto: Revue européenne des sciences socials, 108, pp. 93-106.

ROSE, N. (1991): Governing the Soul: The Shaping of the Private Self, London, Routledge.

ROSE, N. (1998): Inventing Our Selves: Psychology, Power, and Personhood, Cambridge, Cambridge University Press.

SACK, R. D. (1983): «Human territoriality: a theory», Annals of the Association of American Geographers, 73, pp. 55-74.

SACK, R. D. (1986): Human Territoriality: Its Theory and History, Cambridge, Cambridge University Press.

SANDERCOCK, L. (1998): «Introduction: Framing insurgent historiographies for planning», en Sandercock, L. (ed.) Making the Invisible Visible. A Multicultural Planning History, Berkeley, University of California Press.

SEVILLA BUITRAGO, A. (2008): «La ciudad y el eclipse de la experiencia. Notas para una historia crítica de la ordenación territorial», Polis. Revista de la Universidad Bolivariana, v. 7, n. 20, pp. 151-177.

SEVILLA BUITRAGO, A. (2010): «Hacia el origen de la planificación: territorio, enclosure acts y cambio social en la transición del feudalismo al capitalismo», Ciudades, 13, pp. 165-181.

SEVILLA-BUITRAGO, A. (2012): «Territory and the governmentalisation of social reproduction: parliamentary enclosure and spatial rationalities in the transition from feudalism to capitalism», Journal of Historical Geography, 38, pp. 209-219.

SEVILLA-BUITRAGO, A. (2013): «Debating contemporary urban conflicts: A survey of selected scholars», Cities, 31, pp. 454-468.

SOJA, E. W. (1989): Postmodern Geographies: The Reassertion of Space in Critical Social Theory, London, Verso.

EMPIRIA. Revista de Metodología de Ciencias Sociales. N. ${ }^{\circ}$ 27, enero-abril, 2014, pp. 49-72. ISSN: 1139-5737 DOI 10.5944/empiria.27.10862 
THERBORN, G. (1979): ¿Cómo domina la clase dominante?: aparatos de Estado y poder estatal en el feudalismo, el capitalismo y el socialismo, Madrid, Siglo XXI.

URBINATI, N. (1998): «From the Periphery of Modernity: Antonio Gramsci»s Theory of Subordination and Hegemony», Political Theory, 26, pp. 370-391.

WARDE, A. (1985): «Spatial Change, Politics and the Division of Labour», en Derek Gregory \& John Urry (eds.) Social Relations and Spatial Structures, London, MacMillan.

WILLIAMS, G. A. (1960): «The Concept of «Egemonia» in the Thought of Antonio Gramsci: Some Notes on Interpretation», Journal of the History of Ideas, 25, pp. 586-599.

YIFTACHEL, O. (1998): «Planning and Social Control: Exploring the Dark Side», Journal of Planning Literature, 12:4, pp. 395-406. 